Journal of Mathematics and Statistics 5 (4): 369-374, 2009

ISSN 1549-3644

(C) 2009 Science Publications

\title{
On Sixtic Lacunary Spline Solutions of Second Order Initial Value Problem
}

\author{
Jwamer Karwan Hama Faraj \\ Department of Mathematics, College of Science, University of Sulaimani, Iraq
}

\begin{abstract}
Problem statement: The lacunary problem, which we had investigated in this study, consider in finding the spline function of degree six $\mathrm{S}(\mathrm{x})$ of deficiency four, interpolating data given on the function value and third, forth order in the interval $[0,1]$. Also, on the extra initial condition was prescribed on the first derivative. Other purpose of this construction was to solve the second order initial value problem by one example showed that the spline function being interpolation very well compare to [1]. The convergence analysis and the stability of approximation solution were investigated and compared with the exact solution to demonstrate the prescribed lacunary spline $(0,3,4)$ function interpolation. Approach: An approximation with spline functions of degree six and deficiency four is developed for solving initial value problems, with prescribed nonlinear endpoint conditions. Under suitable assumptions with applications showed this spline of the type $(0,3,4)$ are existences, uniqueness and error bounds of the deficient of the solution. Result: Numerical example showed that the presented spline function their effectiveness in solving the second order initial value problem and also showed that our result more well to result in [1]. Also, we note that, the better error bounds were obtained for small step size h. Conclusion: In this study we showed that the lacunary data $(0,3,4)$ are more well approximate to the given second order initial value problem compare with the lacunary data $(0,3,5)$ used in [1].
\end{abstract}

Key words: Spline function, mathematical model, second orders differential equations

\section{INTODUCTION}

The lacunary interpolation problem we investigate in this paper consists in finding the sixtic spline $S(x)$ of deficiency four, interpolating data given on the function and its Cauchy's problem for second derivative at a given set of nodes of the interval [0,1]. The initial value problems play an important role in mathematical physics. As the models considered in applied sciences and engineering are nonlinear in nature, only seldom are analytical solutions available ${ }^{[2]}$. Siddiqi and $\mathrm{Akram}^{[7]}$ used the quintic spline to find approximation solution of fourth order boundary-value problems. In several years various authors have been used spline functions for finding an approximate solution of initial value problem including as ${ }^{[3,4]}$ studies the for third and fourth order boundary value problem.

Consider the second order initial value problem:

$y^{\prime \prime}=f\left(x, y, y^{\prime}\right), y\left(x_{0}\right)=y_{0}, y^{\prime \prime}\left(x_{0}\right)=y_{0}^{\prime \prime}$

And assume that $f \in C^{n-1}\left([0,1] \times R^{2}\right)$ and that $f$ is Lipschitz continuous in $\mathrm{y}$ and $\mathrm{y}^{\prime}$. Spline function been studied by Sallam and Hussien ${ }^{[8]}$ which is used difference way with deficiency three. Uniqueness and error bounded of deficient splines of degree six which is a solution of the lacunary data $(0,1,3$ and 4$)$ has been investigated in ${ }^{[1,5,6]}$.

We approximate the problem (1) by the six degree spline functions as the first boundary condition respect to known third and fourth order derivatives and found the best error bound with convergence analysis.

This study is organized as follows: First consider the spline function of six degree is presented which interpolates the lacunary data $(0,3,4)$. Some theoretical results about existence and uniqueness of the spline function of six degree an introduced and also convergence analysis is studied. To demonstrate the convergence of the prescribed lacunary spline function, numerical example presented, finally we prescribe the conclusion and discussion of the result.

\section{MATERIALS AND METHODS}

Description of the method: we present sixtic spline interpolation for one dimensional and given sufficiently smooth function $\mathrm{f}(\mathrm{x})$ defined on $\mathrm{I}$. And $\Delta_{\mathrm{n}}: 0=\mathrm{x}_{0}<\mathrm{x}_{1}<\mathrm{x}_{2}<\ldots .<\mathrm{x}_{\mathrm{n}}=1$ denote the uniform partition of $\mathrm{I}=[0,1]$ with knots $\mathrm{x}_{\mathrm{i}}=$ ih where $\mathrm{i}=0$, $1,2, \ldots, n$, we denote by $S_{n, 6}^{4}$ the class of sixtic splines $\mathrm{S}(\mathrm{x})$ such that: 


$$
\begin{aligned}
\mathrm{S}_{0}(\mathrm{x})= & \mathrm{y}_{0}+\left(\mathrm{x}-\mathrm{x}_{0}\right) \mathrm{a}_{0,1}+\frac{\left(\mathrm{x}-\mathrm{x}_{0}\right)^{2}}{2} \mathrm{y}_{0}^{\prime \prime}+\frac{\left(\mathrm{x}-\mathrm{x}_{0}\right)^{3}}{6} \mathrm{y}_{0}^{\prime \prime} \\
& +\frac{\left(\mathrm{x}-\mathrm{x}_{0}\right)^{4}}{24} \mathrm{y}_{0}^{(4)}+\left(\mathrm{x}-\mathrm{x}_{0}\right)^{5} \mathrm{a}_{0,5}+\left(\mathrm{x}-\mathrm{x}_{0}\right)^{6} \mathrm{a}_{0,6}
\end{aligned}
$$

On the interval $\left[\mathrm{x}_{0}, \mathrm{x}_{1}\right]$ where $\mathrm{a}_{0, \mathrm{j}}, \mathrm{j}=1,5,6$ are unknowns we need to determine.

And for $\left[\mathrm{x}_{\mathrm{k}}, \mathrm{x}_{\mathrm{k}+1}\right]$ is examine now the inner interval $\Delta_{\mathrm{n}}$. By taking the interpolating conditions, we define $S_{k}(x)$ as:

$$
\begin{aligned}
S_{k}(x)=y_{k} & +\left(x-x_{k}\right) a_{k, 1}+\left(x-x_{k}\right)^{2} a_{k, 2} \\
& +\frac{\left(x-x_{k}\right)^{3}}{6} y_{k}^{\prime \prime \prime}+\frac{\left(x-x_{k}\right)^{4}}{24} y_{k}^{(4)} \\
& +\left(x-x_{k}\right)^{5} a_{k, 5}+\left(x-x_{k}\right)^{6} a_{k, 6}
\end{aligned}
$$

where, $a_{k, j}, k=1(1)(n-2), j=1,2,5,6$ unknowns we need to be determine.

And on the endpoint on the interval can be written $\mathrm{S}_{\mathrm{n}-1}(\mathrm{x})$ in the end of the interval as:

$$
\begin{aligned}
S_{n-1}(x)= & y_{n-1}+\left(x-x_{n-1}\right) a_{n-1,1}+\frac{\left(x-x_{n-1}\right)^{2}}{2} y_{n-1}^{\prime \prime} \\
& +\frac{\left(x-x_{n-1}\right)^{3}}{6} y_{n-1}^{\prime \prime \prime}+\frac{\left(x-x_{n-1}\right)^{4}}{24} y_{n-1}^{(4)} \\
& +\left(x-x_{n-1}\right)^{5} a_{n-1,5}+\left(x-x_{n-1}\right)^{6} a_{n-1,6}
\end{aligned}
$$

The existence and uniqueness theorem for spline function of six degree which interpolated the lacunry data $(0,3,4)$ are presented and examined.

Theorem 1: "Existence and Uniqueness of Spline Function" given the real numbers $\mathrm{y}^{(\mathrm{r})}\left(\mathrm{x}_{\mathrm{i}}\right)$, $r=0,2,3,4$ and $i=0,1,2, \ldots, n$ then there exist a unique spline of degree six as Eq. 2-4 such that:

$\mathrm{S}^{(\mathrm{r})}\left(\mathrm{x}_{\mathrm{i}}\right)=\mathrm{y}^{(\mathrm{r})}\left(\mathrm{x}_{\mathrm{i}}\right)$

Where:

$\mathrm{r}=0,2,3,4$

$\mathrm{i}=0,1,2,3 \ldots, \mathrm{n}$

Proof: let we defined the spline function with respect the interval $[0,1]$ as:

$$
S(x)=\left\{\begin{array}{l}
S_{0}(x) \text { when } x \in\left[x_{0}, x_{1}\right] \\
S_{i}(x) \text { when } x \in\left[x_{i}, x_{i+1}\right], i=1,2, \ldots, n-2 \\
S_{n-1}(x) \text { when } x \in\left[x_{n-1}, x_{n}\right]
\end{array}\right.
$$

And from the conditions (5), can be written Eq. 24 , where the coefficients of these polynomials are to be determined by the following conditions:

$\mathrm{S}_{\mathrm{i}}\left(\mathrm{x}_{\mathrm{i}+1}\right)=\mathrm{S}_{\mathrm{i}+1}\left(\mathrm{x}_{\mathrm{i}+1}\right)=\mathrm{y}_{\mathrm{i}+1}$

$\mathrm{S}_{\mathrm{i}}^{(\mathrm{r})}\left(\mathrm{x}_{\mathrm{i}+1}\right)=\mathrm{S}^{(\mathrm{r})}{ }_{\mathrm{i}+1}\left(\mathrm{x}_{\mathrm{i}+1}\right)=\mathrm{y}_{\mathrm{i}+1}{ }^{(\mathrm{r})}$

Where:

$\mathrm{r}=3,4$

$\mathrm{I}=0,1,2, \ldots, \mathrm{n}-1$

$\mathrm{S}_{\mathrm{i}}^{\prime}\left(\mathrm{x}_{\mathrm{i}+1}\right)=\mathrm{S}_{\mathrm{i}+1}^{\prime}\left(\mathrm{x}_{\mathrm{i}+1}\right)$

and:

$\mathrm{S}_{\mathrm{n}-1}\left(\mathrm{x}_{\mathrm{n}}\right)=\mathrm{y}_{\mathrm{n}}, \mathrm{S}_{\mathrm{n}-1}^{(\mathrm{r})}\left(\mathrm{x}_{\mathrm{n}}\right)=\mathrm{y}_{\mathrm{n}}^{(\mathrm{r})}$

where, $r=3,4$.

To find uniquely the coefficients of Eq. 2 with apply the condition (5) where $\mathrm{i}=0$, we obtain the following:

$$
\begin{aligned}
& h a_{0,1}+h^{5} a_{0,5}+h^{6} a_{0,6}=y_{1}-y_{0}-\frac{h^{2}}{2} y_{0}^{\prime \prime}-\frac{h^{3}}{6} y_{0}^{(3)}-\frac{h^{4}}{24} y_{0}^{(4)} \\
& 60 h^{2} a_{0,5}+120 h^{3} a_{0,6}=y_{1}^{(3)}-y_{0}^{(3)}-h y_{0}^{(4)} \\
& 360 h^{2} a_{0,6}+120 h a_{0,5}=y_{1}^{(4)}-y_{0}^{(4)}
\end{aligned}
$$

And from the boundary condition we have:

$20 h^{3} a_{0,5}+30 h^{4} a_{0,6}=y_{1}^{(2)}-y_{0}^{(2)}-h y_{0}^{(3)}-\frac{h^{2}}{2} y_{0}^{(4)}$

Eliminating these equations we obtain the following:

$$
\left.\begin{array}{rl}
\mathrm{a}_{0,1}= & \frac{1}{\mathrm{~h}}\left[\mathrm{y}_{1}-\mathrm{y}_{0}\right]-\frac{\mathrm{h}}{2} \mathrm{y}_{0}^{\prime \prime}-\frac{\mathrm{h}^{2}}{30}\left[\mathrm{y}_{1}^{(3)}+4 \mathrm{y}_{0}^{(3)}\right] \\
& +\frac{\mathrm{h}^{3}}{120}\left[\mathrm{y}_{1}^{(4)}-2 \mathrm{y}_{0}^{(4)}\right] \\
\mathrm{a}_{0,5}= & \frac{1}{20 \mathrm{~h}}\left[\mathrm{y}_{1}^{(3)}-\mathrm{y}_{0}^{(3)}\right]-\frac{1}{60 \mathrm{~h}}\left[\mathrm{y}_{1}^{(4)}+2 \mathrm{y}_{0}^{(4)}\right] \\
\mathrm{a}_{0,6}= & \frac{1}{120 \mathrm{~h}^{2}}\left[\mathrm{y}_{1}^{(4)}+\mathrm{y}_{0}^{(4)}\right]-\frac{1}{60 \mathrm{~h}^{3}}\left[\mathrm{y}_{1}^{(3)}-\mathrm{y}_{0}^{(3)}\right]
\end{array}\right\}
$$

We shall find the coefficients of $S_{i}(x)$ where $i=1$, 2, 3, ., n-1, from Eq. 6 we obtain:

$$
\left.\begin{array}{l}
h a_{i, 1}+h^{2} a_{i, 2}+h^{5} a_{i, 5}+h^{6} a_{i, 6} \\
=y_{i+1}-y_{i}-\frac{h^{3}}{6} y_{i}^{(3)}-\frac{h^{4}}{24} y_{i}^{(4)} \\
60 h^{2} a_{i, 5}+120 h^{3} a_{i, 6}=y_{i+1}^{(3)}-y_{i}^{(3)}-h y_{i}^{(4)}
\end{array}\right\}
$$


and:

$$
120 \mathrm{~h}^{3} \mathrm{a}_{\mathrm{i}, 5}+360 \mathrm{~h}^{5} \mathrm{a}_{\mathrm{i}, 6}=\mathrm{y}_{\mathrm{i}+1}^{(4)}-\mathrm{y}_{\mathrm{i}}^{(4)}
$$

Eliminating these three equations above us obtain the following:

$$
\begin{aligned}
a_{i, 2}= & -\frac{1}{h} a_{i, 1}+\frac{1}{h^{2}}\left[y_{i+1}-y_{i}\right]-\frac{h}{30}\left[y_{i+1}^{(3)}+4 y_{i}^{(3)}\right] \\
& +\frac{h^{2}}{120}\left[4 y_{i+1}^{(4)}-2 y_{i}^{(4)}\right] \\
a_{i, 5}= & \frac{1}{20 h^{2}}\left[y_{i+1}^{(3)}-y_{i}^{(3)}\right]-\frac{1}{60 h}\left[y_{i+1}^{(4)}+2 y_{i}^{(4)}\right] \\
a_{i, 6}= & \frac{1}{120 h^{2}}\left[y_{i+1}^{(4)}+y_{i}^{(4)}\right]-\frac{1}{60 h^{3}}\left[y_{i+1}^{(3)}-y_{i}^{(3)}\right]
\end{aligned}
$$

Substituting the values of $a_{1,2}, a_{i, 5}$ and $a_{i, 6}$ in the (10), we obtain the following relation between $a_{i+1,1}$ and $a_{i, 1}$ where $S_{i}(x)$ where $i=1,2,3, \ldots n-2$ :

$a_{i+1,2}=2 a_{i, 2}+\frac{h}{2}\left[y_{i+1}^{(3)}+y_{i}^{(3)}\right]-\frac{h^{2}}{12}\left[y_{i+1}^{(4)}+y_{i}^{(4)}\right]$

Thus all coefficients are uniquely determined, so the coefficients matrix of the systems (9) and (10) in the unknown $a_{i, 1}$ where $i=1,2,3, \ldots, n-1$ is a non-singular matrix to determine uniquely. Finally, for the end of interval we obtain the following:

From Eq. 4 and the conditions (7) we have:

$$
\begin{aligned}
& h a_{n-1,1}+h^{5} a_{n-1,5}+h^{6} a_{n-1,6}=y_{n}-y_{n-1} \\
& -\frac{h^{2}}{2} y_{n-1}^{\prime \prime}-\frac{h^{3}}{6} y_{n-1}^{(3)}-\frac{h^{4}}{24} y_{n-1}^{(4)} \\
& 60 h^{2} a_{n-1,5}+120 h^{3} a_{n-1,6}=y_{n}^{(3)}-y_{n-1}^{(3)}-h y_{n-1}^{(4)} \\
& 120 h a_{n-1,5}+360 h^{2} a_{n-1,6}=y_{n}^{(4)}-y_{n-1}^{(4)}
\end{aligned}
$$

and:

$$
\begin{aligned}
a_{n-1,1}= & \frac{1}{h}\left[y_{n}-y_{n-1}\right]-\frac{h}{2} y_{n-1}^{\prime \prime}-\frac{h^{2}}{30}\left[y_{n}^{(3)}+4 y_{n-1}^{(3)}\right] \\
& +\frac{h^{3}}{120}\left[y_{n}^{(4)}-2 y_{n-1}^{(4)}\right]
\end{aligned}
$$

Eliminating these equations we see that $a_{n-1, i}$ where $i=1,2,5,6$ are determined nonzero, a unique solution exists, hence the proof of Theorem 1 is complete.

To find error bounds of the spline $\mathrm{S}(\mathrm{x})$ which solution to the (6). We begin by estimating the error in the interpolation. From (6):

$$
S(x)-y(x)=\sum_{i=0}^{n-1} S_{i}(x)-y(x)
$$

To estimate upper bound error it will be enough to estimate each term, by Taylor's formula:

$$
\begin{aligned}
\left|S_{i}(x)-y(x)\right| & =\left|\sum_{m=0}^{6}\left[S_{i}^{(m)}\left(x_{i}\right)-y^{(m)}\left(x_{i}\right)\right] \frac{\left(x-x_{i}\right)^{m}}{m_{*}}\right| \\
& \leq \sum_{\mathrm{m}=0}^{6}\left|S_{i}^{(m)}\left(x_{i}\right)-y^{(m)}\left(x_{i}\right)\right| \frac{h^{m}}{m_{*}}
\end{aligned}
$$

The bounds on the terms on the right-hand side are obtained by Theorem 2 .

Theorem 2: Let $y \in \mathrm{C}^{6}[0,1]$ and $\mathrm{S}(\mathrm{x})$ be a spline function of six degree in previous theorem then for $\mathrm{x} \in\left[\mathrm{x}_{\mathrm{i}}, \mathrm{x}_{\mathrm{i}+1}\right]$ where $\mathrm{i}=0,1,2 \ldots \mathrm{n}-1$ :

$\left\|S_{i}^{(m)}(x)-y_{i}^{(m)}(x)\right\| \leq\left\{\begin{array}{l}3 W_{6}(h) \\ 4 \mathrm{hW}_{6}(h) \\ \frac{5}{2} h^{2} W_{6}(h) \\ h^{3} W_{6}(h)\end{array}\right.$

and:

$$
\left\|S_{i}^{(m)}(x)-y_{i}^{(m)}(x)\right\| \leq\left\{\begin{array}{l}
\frac{85 h^{4}}{360} W_{6}(h) \\
\frac{46 h^{5}}{360} W_{6}(h) \\
\frac{53 h^{6}}{720} W_{6}(h)
\end{array}\right.
$$

To proof this theorem we need the Lemma 1.

Lemma 1: Let:

$y \in C^{6}[0,1]$ then $\left|e_{i+1,2}-2 e_{i, 2}\right| \leq\left\|y_{i}^{(2)}\right\|+\frac{h^{4}}{12} W_{6}(h)$

where, $\quad e_{i, 2}=a_{i, 2}-y_{i}^{\prime} . \quad W_{6}(h)$ denotes the modules of continuity of $\mathrm{y}^{(6)}$ and $\mathrm{x}_{\mathrm{i}} \prec \theta_{1}, \theta_{2} \prec \mathrm{x}_{\mathrm{i}+1}$.

Proof of lemma: if $\mathrm{y} \in \mathrm{C}^{6}[0,1]$ then using Taylor's expansion formula we have:

$$
\begin{aligned}
\mathrm{y}(\mathrm{x})= & \mathrm{y}\left(\mathrm{x}_{\mathrm{i}}\right)+\left(\mathrm{x}-\mathrm{x}_{\mathrm{i}}\right) \mathrm{y}^{\prime}\left(\mathrm{x}_{\mathrm{i}}\right)+\frac{\left(\mathrm{x}-\mathrm{x}_{\mathrm{i}}\right)^{2}}{2} \mathrm{y}^{\prime \prime}\left(\mathrm{x}_{\mathrm{i}}\right) \\
& +\frac{\left(\mathrm{x}-\mathrm{x}_{\mathrm{i}}\right)^{3}}{6} \mathrm{y}^{\prime \prime \prime}\left(\mathrm{x}_{\mathrm{i}}\right)+\ldots .+\frac{\left(\mathrm{x}-\mathrm{x}_{\mathrm{i}}\right)^{6}}{720} \mathrm{y}^{(6)}\left(\theta_{\mathrm{i}}\right)
\end{aligned}
$$


Where:

$$
\mathrm{x}_{\mathrm{i}} \prec \theta_{\mathrm{i}} \prec \mathrm{x}_{\mathrm{i}+1}
$$

Now from Eq. 9 and 12 with apply (13), we obtain:

$$
\begin{gathered}
e_{i+1,2}-2 e_{i, 2}=y_{i}^{(2)}-\frac{h^{4}}{12} y_{i}^{(4)}\left(\theta_{1}\right)+\frac{h^{4}}{12} y_{i}^{(4)} \\
\left(\theta_{2}\right)+\frac{h^{4}}{24} y_{i}^{(4)}\left(\theta_{3}\right)
\end{gathered}
$$

where, $\quad \mathrm{i}=1,2,3, \ldots, \mathrm{n}-1 \quad$ and $\quad \mathrm{x}_{\mathrm{i}} \prec \theta_{\mathrm{s}} \prec \mathrm{x}_{\mathrm{i}+1}$ where, $s=1,2,3,4$.

It's easy the system of Eq. 12 and 13 in the unknown $\mathrm{e}_{\mathrm{i}, 2}, \mathrm{i}=1,2, \ldots, \mathrm{n}-1$ has unique and exist solution so as ${ }^{[5]}$ :

$$
\left|e_{i+1,2}-2 e_{i, 2}\right| \leq\left|y_{i}^{(2)}\right|+\frac{h^{4}}{12}\left|y^{(4)}\left(\alpha_{1}\right)-y^{(4)}\left(\alpha_{2}\right)\right|
$$

where, $x_{i} \prec \alpha_{1}, \alpha_{2} \prec x_{i+1}$.

This is proof Lemma 1.

\section{Proof of Theorem 2: Let:}

$$
\mathrm{x} \in\left[\mathrm{x}_{\mathrm{i}}, \mathrm{x}_{\mathrm{i}+1}\right]
$$

where, $\mathrm{i}=0,1,2 \ldots, \mathrm{n}-1$.

We have from Eq. 3 with apply Taylor's expansion formula we have:

$$
\mathrm{S}_{\mathrm{i}}^{(6)}(\mathrm{x})=720 \mathrm{a}_{\mathrm{i}, 6}
$$

and:

$$
\mathrm{S}_{\mathrm{i}}^{(5)}(\mathrm{x})=120 \mathrm{a}_{\mathrm{i}, 5}-720 \mathrm{ha}_{\mathrm{i}, 6}
$$

so:

$$
\begin{aligned}
\left|S_{i}^{(6)}(x)-y^{(6)}(x)\right|= & \left|720 a_{k, 6}-y^{(6)}(x)\right| \\
= & \left|3 y_{i}{ }^{(6)}\left(\theta_{1}\right)-2 y^{(6)}\left(\theta_{2}\right)-y^{(6)}\left(\theta_{3}\right)\right| \\
& \left|S_{i}^{(6)}(x)-y^{(6)}(x)\right| \leq 3 W_{6}(h)
\end{aligned}
$$

$\mathrm{W}^{6}(\mathrm{~h})$ Denotes the modules of continuity of $\mathrm{y}^{(6)}$.

From Eq. 6 we have:

$$
\left|S_{i}^{(5)}(x)-y^{(5)}(x)\right|=\left|120 a_{k, 5}-y^{(5)}(x)\right|+h\left|720 a_{k, 6}-y^{(6)}(x)\right|
$$

or:

$$
\begin{aligned}
\left|S_{i}^{(5)}(x)-y^{(5)}(x)\right|= & \left|120 a_{k, 5}-y^{(5)}(x)\right| \\
& +h\left|720 a_{k, 6}-y^{(6)}(x)\right| \\
& \leq(h+3 h) W_{6}(h) \\
& \leq 4 \mathrm{hW}_{6}(\mathrm{~h})
\end{aligned}
$$

and we have also $S_{i}^{(4)}\left(x_{i}\right)-y_{i}^{(4)}\left(x_{i}\right)=0$ then:

$$
\begin{aligned}
\left|S_{i}^{(4)}(x)-y^{(4)}(x)\right| & =\left|\int_{x_{i}}^{x}\left(S_{i}^{(5)}(t)-y_{i}^{(5)}(t)\right) d t\right| \\
& \leq \int_{x_{i}}^{x} h W_{6}(h) d t=4 h^{2} W_{6}(h)
\end{aligned}
$$

From Eq. 15 with apply Taylor's expansion formula as:

$$
\begin{aligned}
& y^{(3)}(x)=y^{(3)}\left(x_{i}\right)+\left(x-x_{i}\right) y^{(4)}\left(x_{i}\right)+\frac{\left(x-x_{i}\right)^{5}}{2} y^{(5)} \\
& \left(x_{i}\right)+\frac{\left(x-x_{i}\right)^{6}}{6} y^{(6)}\left(x_{i}\right) \\
& \left|S_{i}^{(3)}(x)-y^{(3)}(x)\right|=\left|\begin{array}{l}
y_{i}^{(3)}+60 h^{4} a_{i, 5}+\frac{h}{2} y_{i}^{(5)}(x)+120 h^{2} y_{i}{ }^{(6)}\left(\beta_{1}\right) \\
-y^{(3)}\left(x_{i}\right)-\left(x-x_{i}\right) y^{(4)}\left(x_{i}\right)+\frac{\left(x-x_{i}\right)^{5}}{2} \\
y^{(5)}\left(x_{i}\right)+\frac{\left(x-x_{i}\right)^{6}}{6} y^{(6)}\left(\beta_{2}\right)
\end{array}\right| \\
& \leq \frac{\mathrm{h}^{2}}{2}\left|120 \mathrm{a}_{\mathrm{i}, 5}-\mathrm{y}^{(5)}\left(\mathrm{x}_{\mathrm{i}}\right)\right|+ \\
& \frac{\mathrm{h}^{3}}{6}\left|\mathrm{y}_{\mathrm{i}}^{(6)}\left(\beta_{1}\right)-\mathrm{y}^{(6)}\left(\beta_{2}\right)\right| \leq \mathrm{h}^{3} \mathrm{~W}_{6}(\mathrm{~h})
\end{aligned}
$$

where, $x_{i} \prec \beta_{1}, \beta_{2} \prec x_{i+1}$.

It's clear to show that:

$$
\begin{aligned}
\left|S_{i}^{(2)}(x)-y^{(2)}(x)\right| & =\left|y_{i}^{\prime \prime}-2 a_{k, 2}\right|+\frac{h^{3}}{6} \\
& \left|y_{i}^{(5)}-120 a_{k, 5}\right|+\frac{h^{4}}{120}\left|y_{i}^{(6)}-720 a_{k, 6}\right|
\end{aligned}
$$

so:

$\left|\mathrm{y}_{\mathrm{i}}^{\prime \prime}-2 \mathrm{a}_{\mathrm{k}, 2}\right| \leq \frac{2}{45} \mathrm{~h}^{4} \mathrm{~W}_{6}(\mathrm{~h})$ 


$$
\begin{aligned}
\left|S_{i}^{(2)}(x)-y^{(2)}(x)\right|= & \left|y_{i}^{\prime \prime}-2 a_{k, 2}\right|+\frac{h^{3}}{6}\left|y_{i}^{(5)}-120 a_{k, 5}\right| \\
& +\frac{h^{4}}{120}\left|y_{i}^{(6)}-720 a_{k, 6}\right| \\
& \leq h^{4}\left(\frac{2}{45}+\frac{1}{6}+\frac{1}{40}\right) W_{6}(h) \\
& \leq \frac{85}{360} h^{4} W_{6}(h)
\end{aligned}
$$

$$
\left\|S_{0}^{(\mathrm{r})}(\mathrm{x})-\mathrm{y}_{0}^{(\mathrm{r})}(\mathrm{x})\right\| \leq\left\{\begin{array}{l}
\frac{7 \mathrm{~h}^{4}}{24} \mathrm{~W}_{6}(\mathrm{~h}) \\
\frac{26 \mathrm{~h}^{5}}{360} \mathrm{~W}_{6}(\mathrm{~h}) \\
\frac{13 \mathrm{~h}^{6}}{720} \mathrm{~W}_{6}(\mathrm{~h})
\end{array}\right.
$$

Proof:

and we have:

$$
\begin{aligned}
\left|S_{i}^{\prime}(x)-y^{\prime}(x)\right|= & \left|y_{i}^{\prime}-a_{k, 1}\right|+h\left|y_{i}^{\prime \prime}-a_{k, 2}\right|+\frac{h^{4}}{24} \\
& \left|y_{i}^{(5)}-120 a_{k, 5}\right|+\frac{h^{5}}{120}\left|y_{i}^{(6)}-720 a_{k, 6}\right| \\
& \leq h^{5}\left(\frac{2}{45}+\frac{1}{60}+\frac{1}{40}+\frac{1}{24}\right) W_{6}(h) \\
& \leq \frac{46}{360} h^{5} W_{6}(h)
\end{aligned}
$$

Similar way to find $\left|S_{i}(x)-y_{i}(x)\right|$ we obtain:

$$
\begin{aligned}
& \left|S_{i}(x)-y(x)\right| \leq h\left|a_{i, 1}-y^{\prime}\left(x_{i}\right)\right|+\frac{h^{2}}{2}\left|2 a_{i, 2}-y^{\prime \prime}\left(x_{i}\right)\right| \\
& +\frac{h^{5}}{120}\left|120 a_{i, 5}-y^{(5)}\left(x_{i}\right)\right|+\frac{h^{6}}{720}\left|720 a_{i, 6}-y^{(6)}\left(x_{i}\right)\right| \\
& \rightarrow\left|S_{i}(x)-y(x)\right| \leq \frac{h^{6}}{60} W_{6}(h)+\frac{2 h^{6}}{45} W_{6}(h) \\
& +\frac{h^{6}}{120} W_{6}(h)+\frac{h^{6}}{240} W_{6}(h)=\frac{53 h^{6}}{720} W_{6}(h) \\
& \rightarrow\left|S_{i}(x)-y(x)\right| \leq \frac{53 h^{6}}{720} W_{6}(h)
\end{aligned}
$$

This is proof theorem 2 .

Theorem 3: Let $y \in \mathrm{C}^{6}[0,1]$ and $\mathrm{S}(\mathrm{x})$ be a spline function of six degree in previous theorem then for $\mathrm{x} \in\left[\mathrm{x}_{0}, \mathrm{x}_{1}\right]$ :

$$
\left\|S_{0}^{(\mathrm{r})}(\mathrm{x})-\mathrm{y}_{0}^{(\mathrm{r})}(\mathrm{x})\right\| \leq\left\{\begin{array}{l}
3 \mathrm{~W}_{6}(\mathrm{~h}) \\
4 \mathrm{hW}_{6}(\mathrm{~h}) \\
\frac{5 \mathrm{~h}^{2}}{2} \mathrm{~W}_{6}(\mathrm{~h}) \\
\mathrm{h}^{3} \mathrm{~W}_{6}(\mathrm{~h})
\end{array}\right.
$$

and:

$$
\mathrm{S}_{0}^{(6)}(\mathrm{x})=720 \mathrm{a}_{0,6}
$$

so:

$$
\begin{aligned}
& \left|S_{0}^{(6)}(x)-y^{(6)}(x)\right|=\left|720 a_{0,6}-y^{(6)}(x)\right| \leq 3 W_{6}(h) \\
& \left|S_{0}^{(6)}(x)-y^{(6)}(x)\right| \leq 3 W_{6}(h)
\end{aligned}
$$

Similar we obtain:

$\left|S_{0}^{(5)}(x)-y^{(5)}(x)\right| \leq 4 \mathrm{hW}_{6}(\mathrm{~h}),\left|S_{0}^{(4)}(\mathrm{x})-\mathrm{y}^{(4)}(\mathrm{x})\right| \leq \frac{5 \mathrm{~h}^{2}}{2} \mathrm{~W}_{6}(\mathrm{~h})$ $\left|S_{0}^{(3)}(x)-y^{(3)}(x)\right| \leq h^{3} W_{6}(h),\left|S_{0}^{(2)}(x)-y^{(2)}(x)\right| \leq \frac{7 h^{4}}{24} W_{6}(h)$

But for the first derivative and the function we have:

$$
\begin{aligned}
& \left|S_{0}^{\prime}(x)-y^{\prime}(x)\right| \leq\left|a_{0,1}-y^{\prime}\left(x_{0}\right)\right|+\frac{h^{4}}{24}\left|120 a_{0,5}-y^{(5)}\left(x_{0}\right)\right| \\
& +\frac{h^{5}}{120}\left|720 a_{0,6}-y^{(6)}\left(x_{0}\right)\right| \\
& \rightarrow\left|S_{0}^{\prime}(x)-y^{\prime}(x)\right| \leq \frac{h^{5}}{180} W_{6}(h)+\frac{h^{5}}{24} W_{6}(h) \\
& +\frac{h^{5}}{40} W_{6}(h)=\frac{26 h^{5}}{360} W_{6}(h)
\end{aligned}
$$

And for the error bounds of the function with spline function:

$$
\begin{aligned}
& \left|S_{0}(x)-y(x)\right| \leq h\left|a_{0,1}-y^{\prime}\left(x_{0}\right)\right|+\frac{h^{5}}{120}\left|120 a_{0,5}-y^{(5)}\left(x_{0}\right)\right| \\
& +\frac{h^{6}}{720}\left|720 a_{0,6}-y^{(6)}\left(x_{0}\right)\right| \\
& \rightarrow\left|S_{0}(x)-y(x)\right| \leq \frac{h^{6}}{180} W_{6}(h)+\frac{h^{6}}{120} W_{6}(h) \\
& +\frac{h^{6}}{240} W_{6}(h)=\frac{13 h^{6}}{720} W_{6}(h)
\end{aligned}
$$

Similarly we can show that for the interval $\left[\mathrm{x}_{\mathrm{n}-1}, \mathrm{x}_{\mathrm{n}}\right]$ as the initial interval. 
J. Math. \& Stat., 5 (4): 369-374, 2009

Table 1: An absolute maximum error for $\mathrm{S}(\mathrm{x})$ and its derivative order 6 for given problem

\begin{tabular}{lllll}
\hline $\mathrm{h}$ & $\|\mathrm{S}(\mathrm{x})-\mathrm{y}(\mathrm{x})\|_{\infty}$ & $\left\|\mathrm{S}^{\prime}(\mathrm{x})-\mathrm{y}^{\prime}(\mathrm{x})\right\|_{\infty}$ & $\left\|\mathrm{S}^{\prime \prime}(\mathrm{x})-\mathrm{y}^{\prime \prime}(\mathrm{x})\right\|_{\infty}$ & $\left\|\mathrm{S}^{\prime \prime \prime}(\mathrm{x})-\mathrm{y}^{\prime \prime \prime}(\mathrm{x})\right\|_{\infty}$ \\
\hline 0.1 & $1.2 \times 10^{-10}$ & $4 \times 10^{-9}$ & $1.8 \times 10^{-3}$ & $4.25 \times 10^{-6}$ \\
0.2 & $9.1 \times 10^{-8}$ & $7.2 \times 10^{-3}$ & $1.9 \times 10^{-5}$ & $6.9 \times 10^{-8}$ \\
\hline
\end{tabular}

Table 2: Absolute maximum error for $\mathrm{S}(\mathrm{x})$ and its derivative order 6 for given problem

\begin{tabular}{llll}
\hline $\mathrm{h}$ & $\left\|\mathrm{S}^{(4)}(\mathrm{x})-\mathrm{y}^{(4)}(\mathrm{x})\right\|_{\infty}$ & $\left\|\mathrm{S}^{(5)}(\mathrm{x})-\mathrm{y}^{(5)}(\mathrm{x})\right\|_{\infty}$ & $\left\|\mathrm{S}^{(6)}(\mathrm{x})-\mathrm{y}^{(6)}(\mathrm{x})\right\|_{\infty}$ \\
\hline 0.1 & $4.8 \times 10^{-5}$ & $5.1 \times 10^{-3}$ & $5.19 \times 10^{-3}$ \\
0.2 & $5.8 \times 10^{-8}$ & $3.7 \times 10^{-3}$ & $11.5 \times 10^{-2}$ \\
\hline
\end{tabular}

\section{RESULTS}

We present numerical results to demonstrate the convergence of the spline $(0,3,4)$ function of degree six which constructed before to the second order initial value problem.

Problem: Consider the second order initial value problem:

$$
y^{\prime \prime}=\frac{1}{2}\left(y^{\prime}+y\right) \text { when } x \in[0,1], y(0)=y^{\prime \prime}(0)=1
$$

as $^{[1,8]}$ who's the exact solution is $y(x)=e^{x}$, from Eq. 2 it's clear to verify that the spline function on $\left[\mathrm{x}_{0}, \mathrm{x}_{1}\right]$ and all other interval satisfies (6).And compare our result in Table 1 and 2 for example 1 in [1] we conclude that our result more well with [1].

\section{DISCUSSION}

Before proceed with discussion of the numerical results, in this paper have also been treated in an paper [1]. While solving second order initial value problem in that paper, a continuous from of invariant imbedding has been employed, after removing the boundary conditions, which has been solved with step size $h$.

\section{CONCLUSSION}

The numerical example for different values of mesh size $h$ is presented in Table 1 and 2. These approximations corresponds to the iteration index $\mathrm{i}$ by taking $\mathrm{y}_{0}=\mathrm{y}(0)=0$ as the initial approximation. We see that the absolute error criterion $\left|y_{i}^{(r+1)}-y_{i}^{(r)}\right| \leq 10^{-8}$ for all $\mathrm{I}$ is appeared and various this value with respect to $\mathrm{r}$.

As is evident from the numerical results, the method gives $\mathrm{O}\left(\mathrm{h}^{6}\right)$ accuracy, spline solution has its own advantages, once the solution has been computed; the information required for spline interpolation between mesh points is available and which unknown derivative found by the method are better close to the exact solution. Also, may be use the same technique in my paper but for different lacunary data and may be determine which type of lacunary data best approximation to initial value problem.

\section{REFERENCES}

1. Al-Bayati, A.Y., R.K. Saeed and K.H. Faraidun, 2009. The existence, uniqueness and errors bounds for approximation splines interpolation for solving second-order initial value problems. J. Math. Stat., 5: 123-129.

2. Saxena, A. and E. Venturino, 1994. Solving twopoint boundary value problems by means of deficient quartic splines. Applied Math. Comput., 66: 25-40. http://portal.acm.org/citation.cfm?id=202318

3. Ravi Kanth, A.S.V. and Vishnu Bhattacharya, 2006. Cubic spline for a class on non-linear singular boundary value problems arising in physiology. Applied Math. Comput., 174: 768-774. DOI: 10.1016/j.amc.2005.05.022

4. Khan, A. and T. Aziz, 2003. The numerical solution of third-order boundary value problems using quintic spline. Applied Math. Comput., 137: 253-260.

http://portal.acm.org/citation.cfm?id=640238

5. Gary H. and A.K. Varma, 1989. Best error bounds for quartic spline interpolation. Approx. Theor., 58: 58-67.

http://portal.acm.org/citation.cfm?id=69421

6. Saeed, R.K., 1993. (0, 1, 3, 5) Lacunary interpolation by deficient splines. J. Sci. Nat., 2: 28-30.

7. Siddiqi, S.S. and G. Akram, 2003. Quintic spline solutions of fourth order boundary value problem. arXiv. Num. Anal., 1: 1-12. http://arxiv.org/abs/math/0306357

8. Sallam, S. and M.A. Hussien, 1984. Deficient spline function approximation to second-order differential equations. Applied Math. Model., 6: 408-412. DOI: 10.1016/0307-904X(84)90046-5 